\title{
The Impact of the Epidemic on the Macro Economy and Sectoral Industries and Countermeasures
}

\author{
Taeuma Anarwal \\ tarunimaagarwal@gmail.com
}

\begin{abstract}
COVID-19 pandemic has impacted the human lives and economies in an unprecedented manner. There are some sectors which have seen upward growth trajectory and contributed to the ongoing pandemic response at the same time. COVID-19 acted as a boost for fostering Make in India and further strengthening India's roleas a global hub for production of vaccines, PPEs, medicines and masks. Healthcare infrastructure strengthening saw a new commitment from the highest levels of the government. $E$ - commerce is another major sector that has seen a boost and consumers have quickly responded to the changed circumstances and the new normal. Online education in the education industry has witnessed digitization at an unforeseen pace in schools all over the country, with enabling provisions by relevant ministries. The pandemic has therefore proven that in select sectors, India has turned the adversities into opportunities to emerge stronger in select sectors and that has been a silver lining for some!
\end{abstract}

Keywords: COVID-19, economy, India, vaccines, education, E-commerce, manufacturing

\section{Introduction}

COVID-19 pandemic has impacted the human lives in an unprecedented manner as well as on the economies in countries. The economy would take time for its revival. However, there are some sectors which have seen upward growth trajectory and contributed to the ongoing pandemic response at the same time. These have been manifested largely due to multitude of factors and developments such as strengthening of healthcare system, use of digital tools in education, surge in digital literacy, reorganization in companies, digitalization of processes, expansion of e commerce, increase in digital payments and growth in internet based entertainment industry.

\section{Pharmaceuticals Industry - Fostering Make in India}

India a Global Hub for COVID 19 vaccine production, medicines, masks and PPEs for national and international markets

The Indian pharmaceutical industry is the third largest in the world in terms of volume and tenth largest in terms of value. India is one of the biggest suppliers of low - cost pharmaceuticals and vaccines in the world. India is known aptly as the 'pharmacy of the world'. The Indian vaccine market, which has carved out a place for itself at the global level, is expected to reach a valuation of Rs 252 billion by 2025. The Indian market size was Rs 94 billion in 2019. Domestic production of masks and PPE kits has seen an exponential rise also sustaining livelihoods during the COVID 19 pandemic in India - India is exporting masks and PPE kits to other countries after scaling up production. Several medicines such hydroxycholoroquine for COVID 19 treatment have also been exported in the past months all over the world by India.

India is the vaccine manufacturing hub for global vaccine production and supply. Two COVID 19 vaccine candidates in India (Covishield and Covaxin), out of a total 11 worldwide that have received the authorization are being deployed in the vaccine roll out in India, considered as the largest vaccination campaign globally. Many other vaccine candidates are likely to enter the market soon as India has a rich pipeline of candidates currently undergoing clinical trials. Indian firms are big suppliers to the UN agencies, accounting for between $60 \%$ and $80 \%$ of the global vaccine supply. Besides mass - manufacture of vaccines, India has also been successful in creating or modifying a whole new range of vaccines indigenously.

Mission COVID Suraksha was announced with a provision of Rs.900 crores to Department of Biotechnology, Government of India for supporting development of a comprehensive ecosystem for enabling the development of a safe, efficacious and affordable vaccine for COVID-19.

Vaccine Maitri is an humanitarian initiative undertaken by the Gov. of India to provide COVID-19 vaccines to countries around the world. The government started providing vaccines from 20 January 2021. As of 9 May 2021, India had delivered around 66.3 million doses of vaccines to 95 countries. Of these, 10.7 million doses were gifted to 47 countries by the Govt. of India. The remaining 54 million were supplied by the Serum Institute of India under its commercial and WHO COVAX (WHO procurement mechanism) obligations.200, 000 doses of COVID-19 vaccines were gifted by India to the UN on 27 March to be distributed to all peacekeeping missions.

CoWin platform is being made open source, available to any and all countries. India adopted a completely digital approach while planning its vaccination strategy and also helps in tracking the usage of vaccination and minimizes the wastage.

Co - WIN is India's vaccination drive that handles overall management of citizen registration, appointment scheduling, vaccinations and certification. The transparent system allows tracking of each dose of vaccine, monitoring of supplies at vaccination facilities to record the demand at a granular level. 
Indian pharmaceuticals import $70 \%$ of its total API needs from China. At this time, Government of India took important steps to mitigate these problems. Government brought incentive package of Rs.13.76 billion for promoting domestic manufacturing of APIs, critical key starting materials, drug intermediates and medical devices. Union cabinet also amended the Foreign Direct Investment policy to allow FDI up to $100 \%$ under the automatic route for manufacturing medical devices, subject to certain conditions. Pharma sector received investment in sterilization devices, car sanitizers, alcohol sanitizers, sterilization units for hospitals etc. during pandemic period. [Error! Bookmark not defined.]

During COVID- 19 crisis, there was urgent need to manufacture and supply specific items like face masks, hand sanitizers, disinfectants, PPE kits, infrared thermometers and scanners, soap dispensers, diagnostic test kits, inhalators, ventilators etc. The Indian industry divresified itself rapidly to the need and became self - reliant in a couple of weeks. MSMEs sector also utilized this opportunity and has played a key role in this and benefitted both - itself and health sector.

Similarly, COVID- 19 pandemic brought ample of opportunities to pharmaceutical biotechnology industry. India is already a manufacturer of about two third of world vaccine supply. It also started research and development of COVID-19 vaccines.
Diversification by industry to meet the demand - supply gap of COVID-19 products in India

The first case of COVID-19 was detected in the country on 30 Jan 2021, and the Indian manufacturers did not have the capacity to produce high quality Personal Protective Equipment (PPE) kits, the one required as Class - 3 protection under ISO 16603 standards. The country was dependent on imports for the PPEs. Government played a key role in this. Ministry of Health and Family Welfare (MoHFW) and Ministry of Textiles (MoT) came together for planning and understanding the gaps in infrastructure, resources, production, quality, supply chain and packaging. The concerted efforts of two ministries along with other government agencies like DRDO, Alternative Energy Promotion Centre (AEPC), Bureau of Indian Standards (BIS) supported in overcoming the hurdles and levelling the gaps. India, which was importing PPE kits became self sufficient in producing PPE kits of WHO quality standards, within a period of 60 days. Within this period, India witnessed 56 times growth in PPE industry and production increased to 4.5 lakh units per day. Again, MSMEs played a key role in this and all the stakeholders turned this crisis into a valuable opportunity, which, not only saved the industry, but also put an example for other manufacturers under Atmanirbhar Bharat Mission.

\section{PPE PRODUCTION IN INDIA PER DAY}

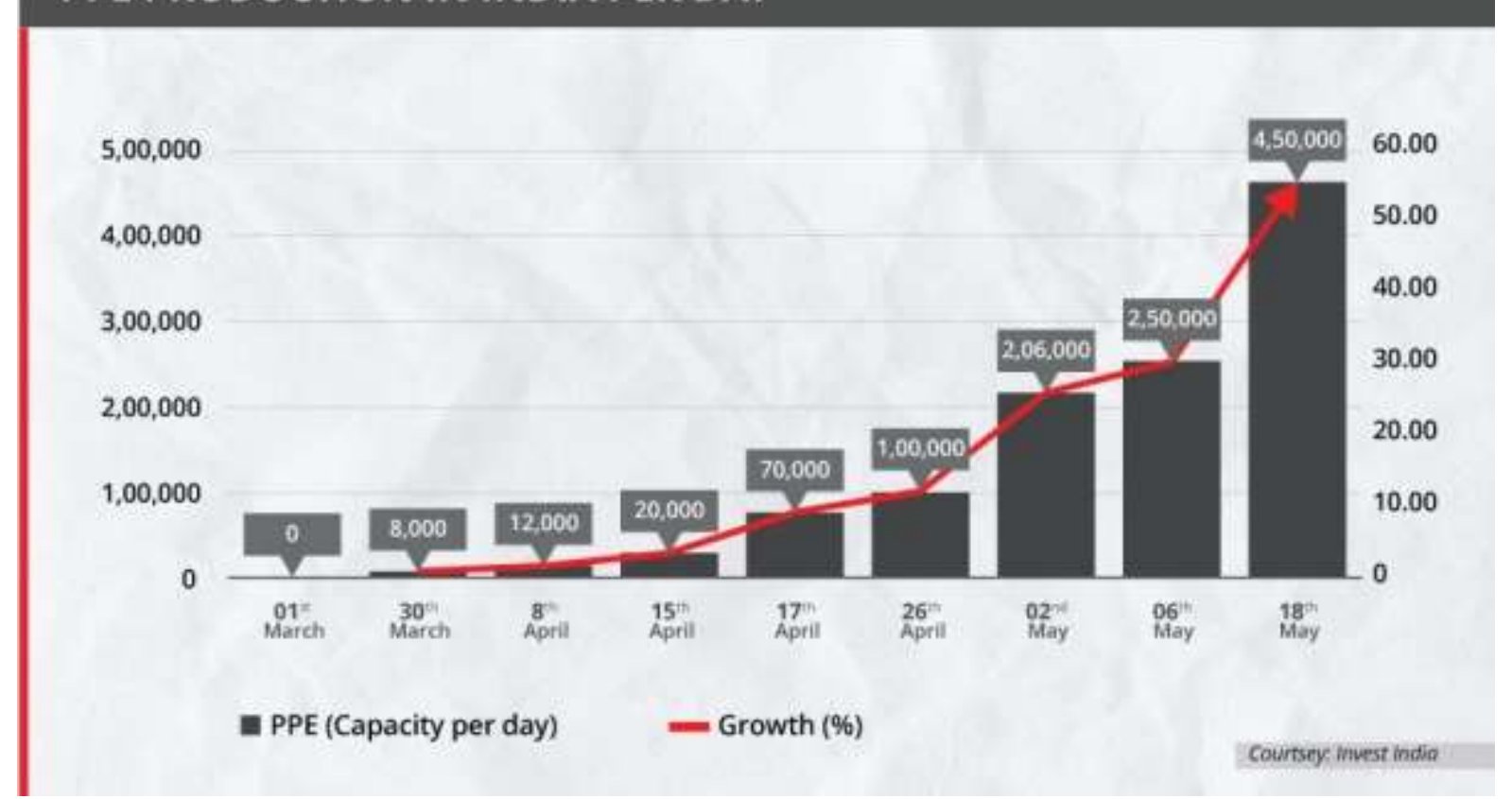

(https: //indiaincgroup. com/india - should - make - ppe - success - a - template - for - all - industries/)

\section{Health Infrastructure Strengthening for Pandemic Response}

The COVID- 19 pandemic brought the importance of healthcare in life and economy of the country. Since the start of the pandemic, healthcare has been center of many policy decisions. The main goal of these policies is to build healthcare infrastructure and skilled manpower, efficient and effective execution, coordination within multiple ministries and between center and states, and bringing public and private sector together.

The healthcare shifted from background to forefront in policies and budgetary outlay of the country. The importance of healthcare can be understood from the fact that the healthcare budget in 2021 was increased by $137 \%$ to 
INR 2.23 crores. The per - capita spending on healthcare has also increased to Rs 1, 944 in 2020 - 21.

Government of India announced PM Aatma Nirbhar Swasth Bharat Yojana with a budget outlay of INR 64, 180 crore over 6 years. Under these schemes, existing national institutions will be strengthened, new institutions will be created and capacities of healthcare systems will be developed.

The main interventions under the scheme are:

1) Support for 17, 788 rural and 11,024 urban Health and Wellness Centers

2) Setting up integrated public health labs in all districts and 3382 block public health units in 11 states;

3) Establishing critical care hospital blocks in 602 districts and 12 central institutions;

4) Strengthening of the National Centre for Disease Control (NCDC), its 5 regional branches and 20 metropolitan health surveillance units;

5) Expansion of the Integrated Health Information Portal to all States/UTs to connect all public health labs;

6) Operationalization of 17 new Public Health Units and strengthening of 33 existing Public Health Units at Points of Entry, that is at 32 Airports, 11 Seaports and 7 land crossings;

7) Setting up of 15 Health Emergency Operation Centers and 2 mobile hospitals; and

8) Setting up of a national institution for One Health, a Regional Research Platform for WHO South East Asia Region, 9

9) Bio - Safety Level III laboratories and 4 regional National Institutes for Virology.

The central and state government announced financial packages and credit incentives to boost the growth of healthcare infrastructure in the country. This is not limited to hospitals but extends to medical equipment and supplies.

Furthermore, many IT projects have been implemented in healthcare sector. Co - WIN app is the most significant example of that, which is the main pillar of COVID-19 vaccination planning and implementation. After about 8.5 months of its launch, it has registered 728 million people and 910 vaccination doses. Almost 50 countries are interested in this application for their own country's vaccination programme.

National Digital Health Mission is working in managing individual health records under unique identity of each individual. Similarly, national health insurance schemes for low - income group like Ayushman Bharat - Pradhan Mantri Jan Arogya Yojana are being executed by National Health Authority.

Moreover, there are multiple reports from states across the country related to various innovations in terms of diagnosis and treatment of COVID-19 disease. It indicates the potential of country's healthcare system which can be utilized for its own strengthening.

\section{Education and the Scaled Up Role of Online Learning}

Education is another domain which underwent rapid transformation due to COVID-19 pandemic and lockdown. Online education tools became the lifeline of education and technological innovations made online learning possible. This is common method which has been adopted by most of the schools to continue education during lock - down. It is based on the availability of computer or mobile phone, an internet connection and an online meeting or learning platform such as zoom, Microsoft Teams, WebEx, Google Meet etc.

Though, EdTech companies got started in the country before the pandemic, they saw exponential growth during lock down. Byzu'shas acquired 7.5 million new users and other companies like Unacademy, Vedantu, and Toppr have also witnessed around 3 times growth. The long - term impact of this transformation is still being debated.

\section{COVID 19 acted as boost for $\mathrm{E}$ - commerce industry across services}

Consumers have quickly responded to the changed circumstances post the COVID-19 outbreak by opting for e commerce services. There has been a shift in consumer behaviour in favour of digital, affecting the purchase decisions. People are embracing digital across entertainment services, health, education, medicines and for deliveries.

The retailers are also bracing to face the changes and are innovating by using $\mathrm{QR}$ codes, providing more digital information at the stores and investing in digital tools.

COVID-19 was a "huge structural boost" for the online pharmacy industry. The pandemic has forced people to adopt a more innovative approaches. This requires behavioural and mindset changes at many levels, including consumers, doctors, health professionals, as well as other stakeholders.

Even many traditional businesses adopted this transformation and fasten digitalization. During the pandemic the online option of sales became the only option for the non - essential retail businesses. But, grocery retail businesses also saw good increase in sales. It is a known fact now that the global retail market hit a slowdown in overall growth. But, an online sale has increased by $18 \%$ compared to last year and went up to was expected to $\$ 3.5$ trillion.

Now retailers are looking out for both, time - tested well known technologies and also the newer ones. These futuristic technologies are - The internet of things (IoT) and artificial intelligence (AI), followed by augmented reality (AR), virtual reality (VR), solutions for digital traceability, robotics, autonomous vehicles (AV) /drones, 3D printing, and blockchain.

As per the GlobalData's recent report, COVID-19 pandemic will continue to have strong positive Impact on E commerce. Because of the lockdown, restriction in 
movement and new living order, every person will have to resort to online purchases and abandon visits to shops.

\section{New users are turning to ecommerce, with long-lasting implications}

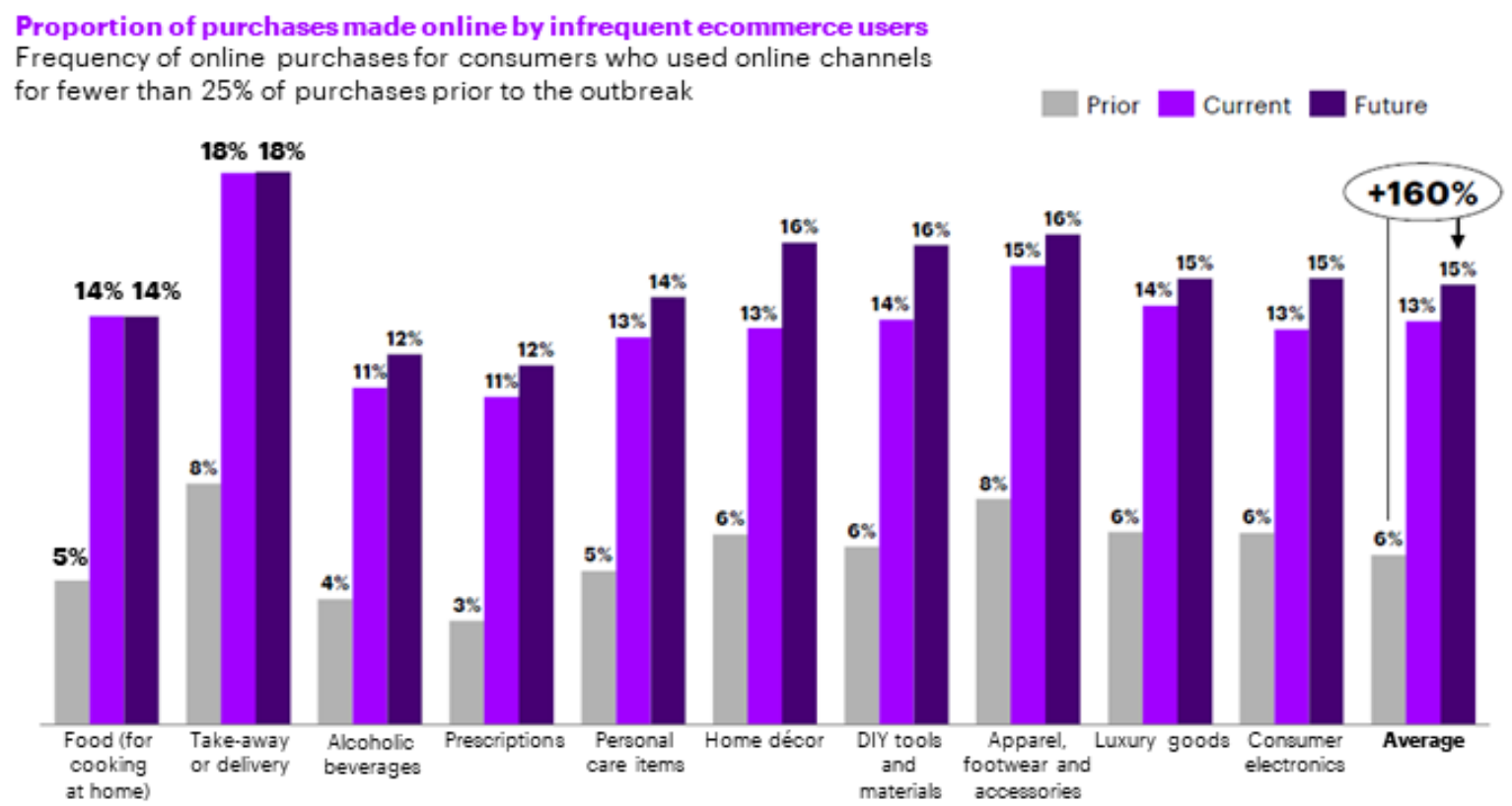

(https: //www.accenture. com/us - en/insights/retail/coronavirus - consumer - behavior - research)

According to a recent report published by Alvarez \& Marsal (Retail Economics and Alvarez \& Marsal), consumers intend to continue online shopping in post - COVID times also.

\section{Summary}

COVID-19 acted as a boost for fostering Make in India and India a global hub for production of vaccines, PPEs, medicines and masks. As part of the 'Vaccine Maitri' programme, India is exporting COVID-19 vaccines to many countries globally. In addition, the masks, PPEs and testing kits are also being exported out of India as a consequence of scaled up production. E - commerce is another major sector that has seen a boost and consumers have quickly responded to the changed circumstances. Online education in the education industry is another major area which has witnessed digitization at an unforeseen pace in schools all over the country, with enabling provisions by relevant ministries. The pandemic has therefore proven that in select sectors, India has turned the adversities into opportunities to emerge stronger and that has been a silver lining for some!

\section{References}

[1] https: //dbtindia. gov. in/pressrelease/department biotechnology - mission - covid - suraksha - supported - biological - e - limited - novel

[2] https: //www.mea. gov. in/vaccine - maitri. htm

[3] https: //health. economictimes. indiatimes. com/news/policy/healthcare - grid - in - india pathway - to - strengthening - health - infrastructure to - support - economic - growth/85072167

[4] Summary of the Budget 2021 - 22, Ministry of Finance. https: //pib. gov. in/PressReleasePage. aspx?PRID=1693908
[5] Sandeep Kumar Mathivananetal. Adoption of E Learning during Lockdown in India. Int J Syst Assur EngManag. 2021 Feb 24: 1-10. Downloaded from https: //www.ncbi. nlm. nih. gov/pmc/articles/PMC7903031/

[6] https: //www.orfonline. org/expert - speak/the - impact - covid19 - pandemic - is - exerting - on - e commerce - 65128/

[7] https: //www.unicef. org/supply/stories/COVID-19 impact - assessment - and - outlook - personal protective - equipment 